\title{
Endometriose, dificuldades no diagnóstico precoce e a infertilidade feminina: Uma Revisão
}

\author{
Endometriosis, difficulties in early diagnosis and female infertility: A review \\ Endometriosis, dificultades en el diagnóstico precoz e infertilidad femenina: Una revisión
}

Recebido: 01/05/2021 | Revisado: 05/05/2021 | Aceito: 06/05/2021 | Publicado: 21/05/2021

Juliana Ilky da Silva Lima Torres

ORCID: https://orcid.org/0000-0002-8064-7036

Universidade Estadual do Maranhão, Brasil

E-mail: julianailky15@hotmail.com

Joabe Lima Araújo

ORCID: https://orcid.org/0000-0002-4806-9192

Universidade de Brasília, Brasil

E-mail: joabearaujobiotec@gmail.com

Julia Augusto Vieira

ORCID: https://orcid.org/ 0000-0003-0566-9603 Universidade de Brasília, Brasil

E-mail: julia.augusto.vieira@gmail.com

Cristiele Dos Santos Souza

ORCID: https://orcid.org/0000-0002-6251-1733

Universidade de Brasília, Brasil

E-mail: cristiele24@gmail.com

Ionara Nayana Gomes Passos

ORCID: https://orcid.org/0000-0003-4729-4977

Universidade Federal do Maranhão, Brasil

E-mail: ionara.passos@gmail.com

Lindalva de Moura Rocha

ORCID: https://orcid.org/0000-0002-5544-8078

Universidade Estadual do Maranhão, Brasil

E-mail: lindalva.nutri.ufpi@gmail.com

\begin{abstract}
Resumo
A endometriose é uma patologia que afeta milhares de mulheres no mundo, isso se deve à presença de tecido endometrial extrauterino, que responde à estimulação hormonal e pode causar uma reação inflamatória resultando em sintomas como dor pélvica crônica severa e infertilidade. No Brasil, cerca de 7 milhões de mulheres sofrem com a doença, cujo diagnóstico, prontuário e pesquisas são altamente deficientes, o que dificulta a recuperação das pacientes. Desse modo, este estudo teve como objetivo investigar as dificuldades no diagnóstico precoce da endometriose e sua relação com a infertilidade feminina por meio de uma revisão narrativa. Foram realizadas buscas bibliográficas nas seguintes bases de dados: SciELO, PubMed/MEDLINE e BVS, por se tratarem de bases de dados que concentram publicações de excelência voltadas para a área da saúde. A seleção dos estudos publicados nas bases de dados foi compreendida entre os anos de 2001 a 2021, utilizando os descritores reconhecidos pelos DECs: "Endometriosis", "Early Diagnosis", "Infertility Female" e "Treatmet Alternatives". Assim, obtivemos resultados de estudos que abordam aspectos envolvidos na dificuldade de se obter o diagnóstico precoce da endometriose, além dos tratamentos disponíveis. Evidenciamos que a endometriose ainda é uma doença de etiopatogenia incerta, e que essa incerteza contribui para a dificuldade de diagnóstico da doença, na qual a falta de informações se destaca como um dos principais fatores que dificultam o diagnóstico precoce da endometriose.
\end{abstract}

Palavras-chave: Endometriose; Diagnóstico precoce; Infertilidade; Tratamento.

\begin{abstract}
Endometriosis is a pathology that affects thousands of women worldwide, this is due to the presence of extrauterine endometrial tissue, which responds to hormonal stimulation and can cause an inflammatory reaction resulting in symptoms such as severe chronic pelvic pain and infertility. In Brazil, about 7 million women suffer from the disease, whose diagnosis, medical records and research are highly deficient, making it difficult for patients to recover. Thus, this study aimed to investigate the difficulties in the early diagnosis of endometriosis and its relation with female infertility through a narrative review. Bibliographic searches were carried out in the following databases: SciELO, PubMed/MEDLINE and BVS, as these are databases that concentrate publications of excellence focused on the health area. The selection of studies published in the databases was understood between the years 2001 to 2021, using the descriptors recognized by the DECs: "Endometriosis", "Early Diagnosis", "Infertility Female" and "Treatmet Alternatives". Thus, we obtained results from studies that address aspects involved in the difficulty of obtaining an early diagnosis of endometriosis, in addition to the available treatments. In which we show that endometriosis is still a
\end{abstract}


disease of uncertain etiopathogenesis, and that this uncertainty contributes to the difficulty of diagnosing the disease, in which one of the biggest factors that hinder the early diagnosis of endometriosis is the lack of information.

Keywords: Endometriosis; Early diagnosis; Infertility; Treatment.

\section{Resumen}

La endometriosis es una patología que afecta a miles de mujeres en todo el mundo, esto se debe a la presencia de tejido endometrial extrauterino, el cual responde a la estimulación hormonal y puede provocar una reacción inflamatoria resultando en síntomas como dolor pélvico crónico severo e infertilidad. En Brasil, alrededor de 7 millones de mujeres padecen la enfermedad, cuyo diagnóstico, historia clínica e investigación son muy deficientes, lo que dificulta la recuperación de los pacientes. Así, este estudio tuvo como objetivo investigar las dificultades en el diagnóstico precoz de la endometriosis y su relación con la infertilidad femenina a través de una revisión narrativa. Se realizaron búsquedas bibliográficas en las siguientes bases de datos: SciELO, PubMed/MEDLINE y BVS, por tratarse de bases de datos que concentran publicaciones de excelencia enfocadas al área de la salud. La selección de estudios publicados en las bases de datos se comprendió entre los años 2001 a 2021, utilizando los descriptores reconocidos por las DEC: "Endometriosis", "Diagnóstico precoz", "Infertilidad femenina" y "Alternativas de tratamiento". Así, obtuvimos resultados de estudios que abordan aspectos implicados en la dificultad de obtener un diagnóstico precoz de endometriosis, además de los tratamientos disponibles. En el que mostramos que la endometriosis sigue siendo una enfermedad de etiopatogenia incierta, y que esta incertidumbre contribuye a la dificultad de diagnóstico de la enfermedad, en el que uno de los mayores factores que dificultan el diagnóstico precoz de la endometriosis es la falta de información.

Palabras clave: Endometriosis; Diagnostico precoz; Esterilidad; Tratamiento.

\section{Introdução}

A endometriose é uma patologia que afeta milhares de mulheres em todo o mundo. Essa patologia ocorre devido à presença de tecido endometrial extrauterino, que responde à estimulação hormonal e pode causar uma reação inflamatória resultando em sintomas como dor pélvica crônica severa e infertilidade (Tomás \& Metello, 2019). A proporção exata de mulheres com a doença ainda é desconhecida, no entanto, os dados mostram que em média 2 a $10 \%$ das mulheres em idade reprodutiva sofrem de endometriose, $3 \%$ das mulheres na pós-menopausa e $40 \%$ das mulheres inférteis também são afetadas pela doença (Borghese et al., 2017; Donatti et al., 2017; Marqui, 2014). No Brasil, os números são igualmente preocupantes. Dados do Ministério da Saúde (MS) apontam que mais de 7 milhões de mulheres têm a doença (Souza, Barros \& Monteiro, 2020), mesmo assim ainda é uma patologia em que o diagnóstico, os registros e as pesquisas são deficientes (Gao et al., 2006).

Isso pode estar relacionado com a incerteza da etiopatogenia da endometriose, embora existam inúmeros estudos de investigação etiológica e patogênica, porém, sabe-se que existe uma combinação de fatores genéticos, imunológicos e hormonais que estimula a formação de surtos ectópicos de endometriose (Kennedy et al., 2005; Sourial, Tempest \& Hapangama, 2014.). Isso reforça a tese de Sampson (1927), que descreve a teoria da implantação, sugerindo que na endometriose ocorra um refluxo do tecido endometrial pelas trompas de falópio durante o período menstrual, bem como posterior implantação e alargamento no peritônio e ovário (Sampson, 1927).

O diagnóstico precoce da endometriose pode proporcionar um tratamento mais eficaz elevando a qualidade de vida da mulher, porém, há dificuldade na identificação dos sintomas e a falta de métodos diagnósticos clínicos especializados, o que contribui para o diagnóstico tardio (Vinatier et al., 2001). Dentre os métodos diagnósticos utilizados, a laparoscopia é considerada o padrão ouro, por ser mais assertiva no estabelecimento do desfecho em adolescentes e adultos (Nogueira et al., 2018; RCOG, 2006).

Mesmo após o diagnóstico e a realização do tratamento, ainda não há confirmação da cura, na qual a paciente não tem certeza se está curada e pode haver recidiva (Denny \& H Mann, 2007). Isso afeta diretamente a sua qualidade de vida, pois a paciente pode sentir dor, ter impacto emocional pela incerteza da sua fertilidade, sentir-se culpada por ter sido vítima de uma doença tão incerta e por não ter nenhuma garantia quanto à realização de novas operações ou tratamentos medicamentos a longo prazo (Novak, 2007). Além disso, a maioria das mulheres que sofrem dessa condição convive com os sintomas por anos sem ter um diagnóstico estabelecido. De acordo com Oliveira et al. (2017), em média, o diagnóstico de endometriose leva 
cerca de 7 a 8 anos desde o início dos sintomas.

Assim, tendo em vista os impactos causados pela endometriose na vida da mulher, principalmente a infertilidade, disfunção sexual, transtornos mentais e diagnósticos tardios, hipotetizamos que esta revisão da literatura científica sobre as possíveis causas que permeiam os atrasos no diagnóstico da Endometriose e sua relação com a infertilidade feminina, possa contribuir para a construção de um melhor cuidado prestado a essas mulheres, permitindo também uma maior compreensão dos aspectos da patologia para ajudar a reduzir os impactos negativos gerados nas mulheres com endometriose. Dessa forma, o objetivo deste estudo foi investigar as dificuldades no diagnóstico precoce da endometriose e sua relação com a infertilidade feminina, a fim de elucidar conhecimentos sobre esses aspectos relacionados à doença, contribuindo para a construção de um melhor atendimento e resolução dos problemas enfrentados por suas portadoras.

\section{Metodologia}

Trata-se de um estudo de revisão narrativa, conforme segue estudos de Bernardo et al. (2004). As bases de dados usadas para as pesquisas bibliográficas foram: Scientific Electronic Library Online (SciELO); National Center for Biotechnology Information (PubMed/MEDLINE) e Biblioteca Virtual em Saúde (BVS). Essas são bases de dados que concentram publicações de excelência voltadas na área da saúde. A seleção dos artigos publicados nas bases de dados foi compreendida entre os anos de 2001 a 2021, utilizando os descritores reconhecidos pelo DECs: "Endometriosis", "Early Diagnosis", "Infertility Female" e "Treatmet Alternatives".

\section{Desenvolvimento}

\subsection{Endometriose}

A endometriose foi descrita pela primeira vez em 1860, na Alemanha, por Rokitansky, ao analisar o material de necropsia e perceber a presença de tecido ectópico semelhante ao tecido endometrial (Bassi, 2011; Barbosa \& de Oliveira, 2015). O autor a definiu como uma patologia ginecológica crônica, que se desenvolve gradativamente e se caracteriza pela presença de tecido endometrial extrauterino, causando dor e intenso fluxo menstrual, tornando, muitas vezes, incapacitante para suas portadoras (São Bento \& Moreira, 2017). É uma das doenças mais comuns, que afetam mulheres em idade reprodutiva, com incidência de 2 a 10\% (Donatti et al., 2017). Apresenta variedade de sintomas, tais como: infertilidade, dor pélvica, dismenorreia e dispareunia. Alguns casos podem ser assintomáticos ou com sintomas de intensidade e localização diferentes, o que dependerá do grau de acometimento da doença (Nogueira et al., 2018; Silva, 2012). Contudo, as regiões da superfície peritoneal, dos ovários, do septo retovaginal, do Sistema Nervoso Central, da pleura e do pericárdio são afetadas mais comumente (Nácul \& Spritzer, 2010).

A endometriose tem afetado com mais frequência mulheres no século XXI e está intimamente relacionada à questão da fertilidade feminina (Caldeira et al., 2017). Estimativas sobre o assunto apontam que, no mundo, 70 milhões de mulheres são acometidas pela patologia, e que se tornou um dos principais motivos de internação por causas ginecológicas nos países industrializados (São Bento \& Moreira, 2017). Em função disso, ficou também conhecida como a "doença da mulher moderna", devido às mudanças nos padrões de vida que sofreram nos últimos anos, em que passaram a ter menos filhos, gravidez tardia, além de adquirir hábitos e estilos de vida que acarretam um maior nível de estresse (Barbosa \& de Oliveira, 2015). Dessa forma, as mulheres passaram a ter menarca precoce, gravidez tardia ou em menor número, o que as tornaram mais suscetíveis ao desenvolvimento da doença, pois esses fatos as levam a ter um maior número de períodos menstruais e, consequentemente, períodos retrógrados (Araújo \& Schmidt, 2020), o que reforça a teoria de Sampson (1927) sobre sua etiologia.

Entre as queixas que assolam as mulheres vítimas de endometriose está a relação com a fertilidade, em que $40 \%$ das 
mulheres com a doença são inférteis, condição que pode ser causada pelas aderências geradas pela patologia bem como pela ineficiência da ovulação e fertilização (Borghese et al., 2017). No entanto, os mecanismos pelos quais a endometriose pode causar infertilidade são controversos, mas acredita-se que possa haver, em parte, relação com o estágio da doença (Silva, 2012). Apesar de ser uma condição que gera grande impacto na vida da mulher, ainda falta acesso aos serviços de diagnóstico e tratamento nas redes de saúde pública e privadas (São Bento \& Moreira, 2017).

\subsection{Aspectos gerais e etiologia}

A etiologia e patogênese da endometriose ainda são incertas. Sabe-se que a formação e o desenvolvimento de focos ectópicos de endometriose podem ter a contribuição da combinação de fatores genéticos, hormonais e imunológicos (Kenned et al., 2005). Mesmo sendo uma doença de etiopatogenia incerta, várias teorias têm sido propostas para esclarecer o aspecto dessas lesões, mas nenhuma delas conseguiu explicar sua etiopatogenia de forma totalmente segura (Bassi, 2011). No entanto, a mais aceita é a Teoria da Menstruação Retrógrada, descrita por Sampsom, em 1927 (Silva, 2012).

Segundo a teoria proposta por este autor, essa condição se deve ao refluxo do tecido endometrial, que ocorre no momento da menstruação, pelas trompas de falópio, com posterior implantação e aumento do peritônio e ovário (Silva, 2012). Mesmo sendo a teoria com maior índice de aceitação, segundo Bricou et al. (2008), de 70 a 90\% das mulheres que apresentam menstruação retrógrada, apenas uma minoria desenvolve a doença, o que evidencia a presença de outros fatores determinantes que estão envolvidos com o seu surgimento, tais como: genéticos, hormonais ou ainda ambientais, segundo Silva (2012). Portanto, até os dias de hoje, ainda não há consenso sobre a etiologia e patogênese da endometriose, mas existem teorias que explicam a ação gerada no organismo que promove o desenvolvimento dessa patologia, considerada multifatorial (Bianco et al., 2010).

\subsection{Epidemiologia}

Dados indicam que, no mundo, existe cerca de 70 milhões de mulheres com endometriose, um dos principais motivos de internação por queixas ginecológicas nos países industrializados (Bellelis et al., 2010). Por ser uma doença dependente de estrogênio, tende a afetar mais as mulheres no período reprodutivo - até $10 \%$ - sendo incomum em outro período - cerca de 3\% das mulheres na pós-menopausa (Barbosa \& de Oliveira, 2015; Donatti et al., 2017; Marqui, 2014). A maioria das portadoras dessa condição começa a ter os primeiros sintomas no início da adolescência, perfazendo um percentual de 40 a 50\% dos casos, mas geralmente o diagnóstico só é estabelecido por volta dos 30 anos (Vigano et al., 2004).

As estimativas da frequência da endometriose mostram que a doença afeta cerca de 15 a $80 \%$ das mulheres com dor pélvica crônica, podendo também afetar 20\% das mulheres na sua forma assintomática (Missmer \& Cramer, 2004). Estudos estatísticos indicam que cerca de 25 a 35\% das mulheres são vítimas de infertilidade, associado a isso, os dados mostram que 30 a 40\% das doentes com endometriose são inférteis (Rampinelli, Milanesi \& Madeira, 2013), o que evidencia a sua estreita relação com a infertilidade feminina.

Outro problema enfrentado é a falta de informação sobre a endometriose pela população em geral, bem como pelos profissionais de saúde. Isso tem favorecido os casos de subdiagnóstico e tem sido um obstáculo para a obtenção de dados epidemiológicos mais precisos sobre a doença (Podgaec, Abrão \& Aldrighi, 2005). Os dados obtidos atualmente são, em sua maioria, extraídos por meio de achados laparoscópicos, enquanto os achados de exames de imagem e diagnóstico clínico são frequentemente negligenciados (Minson et al., 2012).

\subsection{Endometriose no Brasil}

No Brasil, o número de mulheres que sofrem de endometriose é preocupante. Segundo estimativas do MS, cerca de 7 milhões de brasileiras são portadoras da doença, tornando-a um problema de saúde pública devido a sua alta prevalência, 
etiopatogenia indefinida, cronicidade e morbidade (Gomes \& Alves, 2018; Souza, Barros \& Monteiro, 2020; Silva \& de Marqui, 2014). Atingi 15\% das mulheres de 15 a 45 anos, promovendo um percentual de 70\% de mulheres com histórico de nuliparidade, em que mulheres com essa condição têm 20 vezes mais chances de serem inférteis (Borges, 2018).

Tendo em vista o seu grande acometimento no Brasil, o MS deliberou o Protocolo Clínico e as Diretrizes Terapêuticas para o Tratamento da Endometriose, por meio da Portaria SCTIE/MS n ${ }^{\circ}$ 69, de 6 de novembro de 2006, que passou por revisão e atualização posterior pela Portaria SAS/MS n 144, de 31 de março de 2010 (MS, 2006; MS, 2010). Sua finalidade é atuar promovendo a redução da dor causada por esta doença e a eliminação dos surtos endometrióticos no Brasil (Silva \& de Marqui, 2014).

É notório o impacto negativo da doença tanto no sistema de saúde como de forma ainda mais intensa na mulher portadora, prejudicando inclusive sua qualidade de vida, principalmente porque pode causar dores incapacitantes, a presença de infertilidade, bem como a demora e alto custo de exames diagnósticos e para o seu tratamento, que até o momento ainda é inespecífico e apresenta incertezas quanto a recorrência da doença (Denny \& H Mann, 2007; Krina et al., 2020; Koltermann et al., 2016; Hughes et al., 2015).

\subsection{Fatores de risco}

A endometriose é uma condição clínica incerta, apresentando a necessidade de esclarecimento de fatores importantes a seu respeito. Nesta tentativa, desde sua descoberta, estudos têm sido realizados a fim de evidenciar seus fatores de risco, bem como seu desenvolvimento, além de buscar caracterizar de melhor forma essa gama de mulheres acometidas pela patologia. Sabe-se que se trata de uma doença dependente de estrogênio e, por esse motivo, acredita-se que em condições que aumentem a exposição a esse hormônio, haja maior risco para seu surgimento (Gomes \& Alves, 2018). Com isso, sua prevalência torna-se maior em mulheres com menarca precoce, gravidez tardia, que tenham um longo intervalo de tempo entre a menarca e a primeira gravidez e aquelas cuja mãe ou irmã têm a doença, podendo ter seis vezes mais chances de tê-la também (Bellelis et. al., 2010).

Estudos têm demonstrado maior frequência de endometriose em mulheres com melhores condições financeiras. Acredita-se que isso seja resultante do maior acesso dessas mulheres em clínicas de saúde devido a queixas de infertilidade e dores pélvicas, o que aumenta as chances de diagnóstico e obtenção de tratamento. Outra curiosidade é que a endometriose é mais frequentemente identificada em mulheres brancas (Nnoaham et al., 2011).

\subsection{Diagnóstico e tratamento}

O diagnóstico da endometriose ainda é um obstáculo a ser superado, e um dos fatores que contribuem para isso é a diversidade de suas manifestações clínicas que podem ser confundidas com as de outras doenças (Gomes \& Alves, 2018; Silva, 2012). Para a obtenção de um diagnóstico mais preciso, destaca-se a laparoscopia, que é considerada o melhor método, o padrão ouro para diagnósticos de endometriose, por ser mais assertiva em estabelecer o resultado tanto em adolescentes quanto em adultos, permitindo dimensionar e analisar a posição correta dos focos de endometriose, o que gera maior confiabilidade quanto à existência da doença na paciente (Caldeira et al., 2017; Nogueira et al., 2018; RCOG, 2006).

No entanto, não existe um método diagnóstico específico para a detecção de endometriose, mas a ultrassonografia transvaginal e a ressonância magnética nuclear da pelve também são usadas, já que podem mostrar locais da doença avançada e infiltrativa (Nácul \& Spritzer, 2010; Vinatier et al., 2001). Por ser uma doença de difícil diagnóstico, descobri-la torna-se uma sensação de alívio para as portadoras, pois conviver com a doença sem a certeza do diagnóstico é não saber lidar com as dores (Matta \& Muler, 2006).

Mesmo após a obtenção do diagnóstico, ainda não existe um tratamento efetivamente curativo para a endometriose, 
devido à sua etiologia, ainda incerta, e quando a paciente é submetida ao tratamento, ela não tem certeza se está curada podendo haver recidiva. Vale ressaltar que o tratamento aplicado às mulheres acometidas por essa patologia deve ser feito individualmente, considerando aspectos como a profundidade das lesões e se há desejo da mulher em engravidar ou não (Caldeira et al., 2017; Denny e H Mann, 2007; de Souza et al., 2017).

Como alternativas terapêuticas para a patologia pode ser realizado o tratamento medicamentoso, o qual é repassado na maioria das vezes pelos especialistas. Nesse tipo de tratamento, costumam ser utilizados medicamentos para reduzir a quantidade de estrogênio. Pode ser realizado também o tratamento cirúrgico, no caso de mulheres que não tiveram alívio dos sintomas por meio de tratamento medicamentoso. No caso de mulheres com algum problema de fertilidade, tanto o tratamento medicamentoso quanto a cirurgia ou técnicas de reprodução assistida podem ser realizados, visando uma possível gravidez (Caldeira et al., 2017; de Souza et al., 2017).

Os sintomas gerados pela doença afetam as mulheres em diversos aspectos: dificulta o trabalho, a vida social, a fertilidade e prejudica suas emoções devido a difícil trajetória que vai do diagnóstico ao tratamento, além dos elevados custos com saúde, exames e internações, o que resulta em diversos transtornos à paciente, evidenciando a importância do diagnóstico precoce da endometriose como fator essencial para o bom resultado terapêutico e prognóstico (de Souza et al., 2017; Santos et al., 2012).

\section{Considerações finais}

Evidenciamos que a endometriose ainda é uma doença de etiopatogenia incerta, e que a teoria mais aceita até o momento é a do pesquisador Sampson (1927). A falta de informações tem se mostrado o fator que mais contribui para a dificuldade de diagnóstico da endometriose, em que a família e/ou a própria portadora da doença e até mesmo os profissionais da saúde normalizem uma forte cólica vivenciada por uma mulher próxima a seu período menstrual. Assim novos estudos originais que investiguem sua etiopatogenia e estudos de revisões sistemáticas são essenciais, nos quais mais informações sobre a doença possam surgir para que novos tratamentos possam ser desenvolvidos para servir de embasamento para especialistas realizarem investigações mais profundas e atentas como o exame de laparoscopia que, até o momento, é considerado o padrão ouro para o diagnóstico da endometriose em pacientes que apresentam cólicas intensas.

\section{Agradecimentos}

Os autores agradecem a colaboração do Grupo de Pesquisa em Ciências Naturais e Biotecnologia - CIENATEC da Universidade Federal do Maranhão - UFMA, Campus Universitário de Grajaú e a Coordenação de Aperfeiçoamento de Pessoal de Nível Superior - CAPES pela bolsa de estudo.

\section{Referências}

Araújo, F. W. C.; Schmidt, D. B. (2020). Endometriose um problema de saúde pública: revisão de literatura. Revista Saúde e Desenvolvimento, 14(18), 25-37. Barbosa, D. A. de S., \& de Oliveira, A. M. (2015). Endometriose e seu impacto na fertilidade feminina. Saúde \& Ciência Em Ação, 1(1), 43-56.

Bassi, M. A. (2011). Cinética celular na endometriose profunda infiltrativa de reto-sigmoide: estudo anátomo-clínico (Doctoral dissertation, Universidade de São Paulo).: <https://www.teses.usp.br/teses/disponiveis/5/5139/tde-23112011-191633/en.php>.

Bellelis, P., Dias Jr, J. A., Podgaec, S., Gonzales, M., Baracat, E. C., \& Abrão, M. S. (2010). Epidemiological and clinical aspects of pelvic endometriosis: series of cases. Revista da Associacao Medica Brasileira, 56(4), 467-471. http://dx.doi.org/10.1590/S0104-42302010000400022

Bernardo, W. M., Nobre, M. R. C., \& Jatene, F. B. (2004). A prática clínica baseada em evidências: parte II-buscando as evidências em fontes de informação. Revista Brasileira de Reumatologia, 44(6), 403-409. https://doi.org/10.1590/S0482-50042004000600003

Bianco, B., Christofolini, D. M., de Souza, Â. M. B., \& Barbosa, C. P. (2010). O papel dos desreguladores endócrinos na fisiopatologia da endometriose: revisão da literatura. Arquivos Brasileiros de Ciências da Saúde, 35(2), 1-8. https://doi.org/10.7322/abcs.v35i2.95

Borges, A. J. S. (2018). Aspectos atuais da endometriose. Trabalho de conclusão de curso. Universidade Federal do Maranhão. Disponível em: http://hdl.handle.net/123456789/2363. 
Borghese, B., Zondervan, K. T., Abrao, M. S., Chapron, C., \& Vaiman, D. (2017). Recent insights on the genetics and epigenetics of endometriosis. Clinical genetics, 91(2), 254-264. https://doi.org/10.1111/cge.12897

Bricou, A., Batt, R. E., \& Chapron, C. (2008). Peritoneal fluid flow influences anatomical distribution of endometriotic lesions: why Sampson seems to be right. European Journal of Obstetrics \& Gynecology and Reproductive Biology, 138(2), 127-134. https://doi.org/10.1016/j.ejogrb.2008.01.014

Caldeira, T. D. B., Serra, I. D., Inácio, L. D. C., \& Terra, I. B. N. (2017). Infertilidade na endometriose: etiologia e terapêutica. HU Revista, 43(2), 173-178. https://doi.org/10.34019/1982-8047.2017.v43.2677

Da Matta, A. Z., \& Muller, M. C. (2006). Uma análise qualitativa da convivência da mulher com sua endometriose. Psicologia, saúde e doenças, 7(1), 57-72.

De Souza, G. K. T., Costa, J. R. G., de Oliveira, L. L., \& de Lima, L. R. (2017). Endometriose x infertilidade: revisão de literatura. Encontro de Extensão, Docência e Iniciação Científica (EEDIC), 3(1), 1-4.

Denny, E., \& H Mann, M. C. (2007). A clinical overview of endometriosis: a misunderstood disease. British journal of nursing, 16(18), 1112-1116. https://doi.org/10.12968/bjon.2007.16.18.27503

Donatti, L., Ramos, D. G., Andres, M. D. P., Passman, L. J., \& Podgaec, S. (2017). Pacientes com endometriose que utilizam estratégias positivas de enfrentamento apresentam menos depressão, estresse e dor pélvica. Einstein (São Paulo), 15(1), 65-70. https://doi.org/10.1590/s1679-45082017ao3911

Gao, X., Yeh, Y. C., Outley, J., Simon, J., Botteman, M., \& Spalding, J. (2006). Health-related quality of life burden of women with endometriosis: a literature review. Current medical research and opinion, 22(9), 1787-1797. https://doi.org/10.1185/030079906X121084

Gomes, N. A., \& Alves, K. (2018). A ressonância magnética no diagnóstico de endometriose profunda com acometimento intestinal: relato de caso. UNILUS Ensino e Pesquisa, 15(38), 25-39.

Hughes, C. L., Foster, W. G., Agarwal, S. K., \& Mettler, L. (2015). The impact of endometriosis on the health of women. BioMed Research International, 365951.

Kennedy, S., Bergqvist, A., Chapron, C., D’Hooghe, T., Dunselman, G., Greb, R., ... \& Saridogan, E. (2005). ESHRE guideline for the diagnosis and treatment of endometriosis. Human reproduction, 20(10), 2698-2704. https://doi.org/10.1093/humrep/dei135

Koltermann, K. C., Schlotmann, A., Schröder, H., Willich, S. N., \& Reinhold, T. (2016). Economic burden of deep infiltrating endometriosis of the bowel and the bladder in Germany: The statutory health insurance perspective. Zeitschrift für Evidenz, Fortbildung und Qualität im Gesundheitswesen, 118, 24-30. https://doi.org/10.1016/j.zefq.2016.09.006

Krina, T. Z., Christian, M. B., \& Stacey, A. M. (2020). Endometriosis. New England Journal of Medicine, 382, 1244-56.

Marqui, A. B. T. D. (2014). Endometriose: do diagnóstico ao tratamento. Revista de Enfermagem e Atenção à Saúde, 97-105.

Minson, F. P., Abrão, M. S., Sardá Júnior, J., Kraychete, D. C., Podgaec, S., \& Assis, F. D. (2012). Importância da avaliação da qualidade de vida em pacientes com endometriose. Revista Brasileira de Ginecologia e Obstetrícia, 34(1), 11-15. https://doi.org/10.1590/S0100-72032012000100003

Missmer, S. A., \& Cramer, D. W. (2004). METHODOLOGIC ISSUES. Endometriosis in Clinical Practice, 66.

MS - Ministério da Saúde. (2006). 〈http://bvsms.saude.gov.br/bvs/saudelegis/sctie/2006/prt0069_01_11_2006_comp.html>.

MS - Ministério da Saúde. (2010).: 〈http://bvsms.saude.gov.br/bvs/saudelegis/sas/2010/prt0144_31_03_2010.html〉.

Nácul, A. P., \& Spritzer, P. M. (2010). Aspectos atuais do diagnóstico e tratamento da endometriose. Revista Brasileira de ginecologia e obstetrícia, 32(6), 298-307. https://doi.org/10.1590/S0100-72032010000600008

Nnoaham, K. E., Hummelshoj, L., Webster, P., d'Hooghe, T., de Cicco Nardone, F., de Cicco Nardone, C., ... \& Study, W. E. R. F. G. (2011). Impact of endometriosis on quality of life and work productivity: a multicenter study across ten countries. Fertility and sterility, 96(2), 366-373. https://doi.org/10.1016/j.fertnstert.2011.05.090

Nogueira, A. C. R., Santiago, M. T., Bahia, C. P., \& Soares, H. H. P. (2018). Tratamento da endometriose pélvica: uma revisão sistemática. Revista Científica FAGOC-Saúde, 3(2), 38-43.

Novak, E. (2007). Berek \& Novak's gynecology. Lippincott Williams \& Wilkins.

Oliveira, M. A. P., Raymundo, T. S., Soares, L. C., Pereira, T. R., \& Demôro, A. E. (2017). How to use CA-125 More Effectively in the Diagnosis of Deep Endometriosis?. Journal of Minimally Invasive Gynecology, 24(7), S112-S113. https://doi.org/10.1155/2017/9857196

Podgaec, S., Abrão, M. S., \& Aldrighi, J. M. (2005). Aspectos hormonais da endometriose. Endocrinologia ginecológica: aspectos contemporâneos. São Paulo: Atheneu, 221-228.

Rampinelli, H., Milanese, B. C., \& Madeira, K. (2013). Perfil epidemiológico das pacientes atendidas em um consultório privado e submetidas à videolaparoscopia para tratamento de endometriose na região de Criciúma. ACM Arq. Catarin. Med, 09-14.

RCOG - Royal College of Obstetricians and Gynecologists (2006). Endometriosis, Investigation and Management (Green-top Guideline No. 24).

Sampson, J. A. (1927). Metastatic or Embolic Endometriosis, due to the Menstrual Dissemination of Endometrial Tissue into the Venous Circulation. The American journal of pathology, 3(2), 93-110.

Santos, T. M. V., Pereira, A. M. G., Lopes, R. G. C., \& Depes, D. D. B. (2012). Tempo transcorrido entre o início dos sintomas e o diagnóstico de endometriose. Einstein (São Paulo), 10(1), 39-43. http://dx.doi.org/10.1590/S1679-45082012000100009 
São Bento, P. A. D. S., \& Moreira, M. C. N. (2017). A experiência de adoecimento de mulheres com endometriose: narrativas sobre violência institucional. Ciência \& Saúde Coletiva, 22, 3023-3032. https://doi.org/10.1590/1413-81232017229.03472017

Silva, A. D. (2012). Endometriose e infertilidade: o papel do tratamento cirúrgico prévio a ciclos de procriação medicamente assistida (Dissertação de Mestrado) Universidade do Porto. Porto, Portugal. Disponível em: https://sigarra.up.pt/icbas/pt/pub_geral.pub_view?pi_pub_base_id=30741

Silva, M. P. C., \& de Marqui, A. B. T. (2014). Qualidade de vida em pacientes com endometriose: um estudo de revisão. Revista Brasileira em Promoção da Saúde, 27(3), 413-421. http://dx.doi.org/10.5020/18061230.2014.p413

Sourial, S., Tempest, N., \& Hapangama, D. K. (2014). Theories on the pathogenesis of endometriosis. International journal of reproductive medicine, 2014, 179515. https://doi.org/10.1155/2014/179515

Souza, L. G., Barros, A. M. D. A., \& Monteiro, M. R. S. (2020). A importância do CA-125 para o diagnóstico precoce da endometriose. Revista de Patologia do Tocantins, 7(1), 66-70. https://doi.org/10.20873/uft.2446-6492.2020v7n1p66

Tomás, C., \& Metello, J. L. (2019). Endometriose e infertilidade-onde estamos?. Acta Obstétrica e Ginecológica Portuguesa, 13(4), $235-241$.

Viganò, P., Parazzini, F., Somigliana, E., \& Vercellini, P. (2004). Endometriosis: epidemiology and aetiological factors. Best practice \& research Clinical obstetrics \& gynaecology, 18(2), 177-200. https://doi.org/10.1016/j.bpobgyn.2004.01.007

Vinatier, D., Orazi, G., Cosson, M., \& Dufour, P. (2001). Theories of endometriosis. European Journal of Obstetrics \& Gynecology and Reproductive Biology, 96(1), 21-34. https://doi.org/10.1016/S0301-2115(00)00405-X 